\title{
Estilo de vida $e$ intensidade da dor no período folicular
}

\author{
Lifestyle and pain intensity in follicular phase
}

Como citar este artigo:

SANTOS, G. K. A.; BECKER, C. A. S.; SILVA, N. C. O. V.; ALFIERI, F. M. Estilo de vida e intensidade da dor no período folicular. Revista Saúde

(Sta. Maria). 2019; 45 (2).

\section{Autor correspondente:}

Nome: Gyan Karla Advíncola dos

Santos

E-mail: karlaadvincola@hotmail.com Telefone: (65) 9909-2179

Formação Profissional: Mestranda em Promoção da Saúde pelo Centro Universitário Adventista de São Paulo (UNASP), São Paulo, SP, Brasil.

Filiação Institucional: Mestrado Profissional em Promoção da Saúde (UNASP)

Endereço para correspondencia: Estrada de Itapecerica $n^{\circ}: 5859$

Bairro: Jardim IAE Cidade: São

Paulo Estado: São Paulo

CEP: 05858-00 1

Data de Submissão:

26/03/2019

Data de aceite:

04/08/2019

Conflito de Interesse: Não há conflito de interesse

\section{(cc) $\mathrm{B} Y-\mathrm{NC}-\mathrm{ND}$}

\author{
Gyan Karla Advíncola dos Santos, Crislayne Advíncula dos Santos Becker, \\ Natália Cristina de Oliveira Vargas e Silva, Fábio Marcon Alfieri
}

\section{RESUMO}

Objetivo: verificar o objetivo deste estudo foi relacionar a intensidade da dor relatada durante o período folicular com o estilo de vida adotado por universitárias. Métodos: Trata-se de um estudo transversal observacional que envolveu mulheres com idade entre 18 e 30 anos. A avaliação da dor na fase folicular foi feita pela escala visual analógica e o estilo de vida avaliado pelo questionário Estilo de Vida FANTASTICO. As participantes eram universitárias de um Centro Universitário privado localizado na zona sul da cidade de São Paulo e a pesquisa aconteceu nos meses de agosto a dezembro de 2018. As universitárias foram divididas em três grupos segundo a intensidade da dor (0-3,9: leve; 4-7,9: moderada e 8-10: intensa). Para avaliar as associações entre as variáveis foi empregado 0 teste de correlação de Spearman, e as comparações entre os 3 grupos foram realizadas pela análise de variância a um fator (one-way ANOVA), com post hoc de Bonferroni. Resultados: a amostra foi composta por 110 mulheres, com média de idade 20,9 \pm 2 anos e a média de intensidade de dor foi de $5,7 \pm 2,8$ na escala visual analógica. Não houve associação entre a escala visual analógica e o estilo de vida. Contudo, houve diferença significante em relação ao estilo de vida das mulheres com intensidade de dor leve quando comparadas com aquelas com dor moderada e intensa, mostrando que mulheres com melhor EV possuem menor dor referida no período folicular do ciclo menstrual. Conclusões: Não há associação entre estilo de vida e intensidade de dor referida durante o período folicular de universitárias, entretanto ao serem divididas de acordo com a intensidade da dor, observa-se que aquelas com menos dor possuem melhor estilo de vida.

PALAVRAS-CHAVE: Estilo de vida; Dor; Ciclo menstrual.

\section{ABSTRACT}

Objective: To verify the objective of this study was to relate the intensity of pain reported during the follicular period with the lifestyle adopted by university students. Methods: It was an observational cross-sectional study involving university women aged between 18 and 30 years old. Pain in the follicular phase was evaluated by the visual analogue scale and the LS by the FANTASTICO Lifestyle questionnaire. The university students were divided into three groups according to the intensity of the pain (0-3.9: low, 4-7.9: moderate and 8-10: intense). Spearman's correlation test was employed to evaluate the associations between variables, and comparisons between the three groups were performed by one-way ANOVA, with Bonferroni post hoc test. Results: The sample consisted of 110 women, with mean age of $20.9 \pm 2$ years and mean pain intensity of $5.7 \pm 2.8$ in visual analogue scale. There was no association between visual analogue scale and Lifestyle. However, there was a significant difference regarding Lifestyle of women with low pain intensity when compared to those with moderate and intense pain, showing that women with better Lifestyle had lower referred pain in the follicular phase of the menstrual cycle. Conclusions: There is no association between lifestyle and referred pain intensity during the follicular period of college students, however, when divided according to pain intensity, it is observed that those with less pain have a better lifestyle.

KEYWORDS: Life style; Pain; Menstrual cycle. 


\section{INTRODUÇÃO}

A dor pode ser considerada uma experiência não agradável que envolve aspectos sensoriais e emocionais e pode ou não estar relacionada a danos nos tecidos corporais de acordo com a Associação Internacional para Estudos da Dor (IASP) $)^{1}$.

A dor representa um problema de saúde pública, considerando suas consequências físicas e emocionais, que podem resultar em quadros de ansiedade e de incapacidades temporárias ou permanentes ${ }^{2}$. Em relação a prevalência de dor ser maior nas mulheres, esta pode ser decorrente dos fatores hormonais decorrentes do ciclo menstrual ${ }^{2}$.

Os distúrbios menstruais podem influenciar não apenas a fertilidade das adolescentes e mulheres, mas também sua saúde mental e qualidade de vida. Estudos epidemiológicos identificaram alta prevalência de dismenorreia, variando entre $43 \%$ e $91 \%$ em adolescentes, e fatores de estilo de vida (incluindo atividade física e alimentação) estão relacionados à esta condição ${ }^{3}$.

Já foi constatado que durante a fase folicular mulheres com histórico de dismenorreia apresentam maior percepção de dor, resultando em aumento da sensibilidade dolorosa e prejuízo em alguns aspectos hemodinâmicos em repouso e durante o exercício, bem como na aptidão cardiorrespiratória ${ }^{4}$. O cuidado com o estilo de vida e com a saúde geral são essenciais para o manejo dos sintomas menstruais ${ }^{5}$.

Segundo a Organização Mundial da Saúde, Estilo de Vida (EV) "é o conjunto de hábitos e costumes que são influenciados, modificados, encorajados ou inibidos pelo prolongado processo de socialização"6. Esses hábitos e costumes incluem o uso de substâncias como álcool, fumo, chá ou café, hábitos dietéticos e de exercício. Eles têm importantes implicações para a saúde e são frequentemente objeto de investigações epidemiológicas ${ }^{6}$.

Um estilo de vida saudável é um modo de vida que sustenta e melhora a saúde e o bem-estar. Mais importante, envolve uma dieta saudável, atividade física regular, formas de lidar com o estresse, comunicação interpessoal e responsabilidade com a saúde ${ }^{7}$. O estilo de vida inclui comportamentos controláveis e mutáveis que podem afetar a saúde do indivíduo de forma positiva ou negativa ${ }^{7}$. Entendendo que a intensidade da dor durante a fase folicular do ciclo menstrual pode estar associada ao estilo de vida adotado pelas mulheres, hipotetizamos neste estudo que existe relação entre a menor percepção de dor e melhor estilo de vida entre mulheres universitárias, o objetivo deste estudo foi relacionar a intensidade da dor relatada durante o período folicular com o estilo de vida adotado por universitárias.

\section{MÉTODO}

Este estudo foi aprovado pelo Comitê de Ética em Pesquisa do Centro Universitário Adventista de São Paulo 
(parecer número 2.141.655) e conduzido de acordo com as normas da Resolução 466 do Conselho Nacional de Saúde.

Duzentas e cinquenta estudantes universitárias foram contatadas para participar do estudo. Destas, 181 consentiram a participação, contudo, 71 responderam ao questionário sobre estilo de vida, Estilo de Vida Fantástico de forma incompleta, e por esta razão foram excluídas da análise.

Trata-se de um estudo transversal observacional do qual participaram 110 universitárias com idade entre 18 a 30 anos, recrutadas por conveniência entre as estudantes de um Centro Universitário privado localizado na zona sul da cidade de São Paulo. A pesquisa aconteceu nos meses de agosto a dezembro de 2018. O tamanho da amostra foi baseado no estudo de Quintana et. al. ${ }^{8}$ que tiveram como objetivo verificar a influência do nível de atividade física sobre a dismenorreia em 100 mulheres universitárias.

Foram excluídas da amostra gestantes, tabagistas, mulheres que possuíam filhos, histórico de doenças uterinas e as que faziam uso de contraceptivos hormonais. Estes critérios foram adotados baseado no estudo de Quintana et. al. ${ }^{8}$ pois poderiam interferir nos resultados obtidos.

Para avaliar o estilo de vida, foi empregado o questionário Estilo de Vida FANTASTICO, validado na versão em português para o Brasil ${ }^{9}$. O instrumento considera o comportamento dos indivíduos no último mês, e seus resultados permitem determinar a associação entre o estilo de vida e a saúde. Ele possui 25 questões distribuídas em nove domínios (família e amigos; atividade física; nutrição; cigarro e drogas; álcool; sono, cinto de segurança, estresse e sexo seguro; tipo de comportamento; introspecção; trabalho). As questões estão dispostas na forma de escala Likert e a pontuação total varia de 0 a 100 pontos, quanto maior, melhor o estilo de vida.

Para avaliar a intensidade da dor referida durante a última a fase folicular do ciclo menstrual, foi usada a Escala Visual Analógica $(E V A)^{10}$, que consiste em uma reta de $10 \mathrm{~cm}$, onde 0 representa ausência de dor, e 10 representa dor insuportável. Ambas as avaliações foram feitas não importando o período do ciclo menstrual, porém lembrando a participante de ter em mente a última fase folicular do seu ciclo menstrual.

A análise dos dados foi feita no pacote estatístico SPSS v.24 para Windows. Os dados foram apresentados como médias e desvios-padrão. Para avaliar as associações entre as variáveis foi empregado o teste de correlação de Spearman (após o teste de normalidade de Kolmogorov-Smirnov rejeitar a distribuição normal dos dados). Os resultados foram classificados como correlação muito fraca $(r=0,00$ a 0,19$)$, fraca $(r=0,20$ a 0,39$)$, moderada $(r=0,40$ a 0,59$)$, forte $(r=0,60$ a 0,79$)$ e muito forte $(r=0,80$ a 1,00). Em todos os casos, o nível descritivo a estabelecido foi de $5 \%$. Por fim, as comparações entre os 3 grupos de intensidade de dor avaliada pela EVA (0-3,9: leve; 4-7,9: moderada e 8-10: intensa) ${ }^{10}$ foram realizadas pela análise de variância a um fator (one-way ANOVA), com post hoc de Bonferroni. 


\section{RESULTADOS}

Assim, a amostra deste estudo foi composta por 110 mulheres com média de idade de 20,94 $\pm 2,03$ anos, e Índice de Massa Corporal (IMC) de 22,99 $\pm 4,08 \mathrm{~kg} / \mathrm{m}^{2}$, o que as classifica, em média, como eutróficas. A média do escore da EVA reportada durante o período folicular foi considerada moderada, $5,72 \pm 2,79 \mathrm{~cm}$.

A percepção de dor não se associou com o EV ( $r=-0,16, p=0,102)$, nem tampouco com o IMC $(r=-0,06, p=0,544)$. Quando a amostra foi dividida em três grupos de acordo com a intensidade da dor avaliada pela EVA (G1: 0,0-3,9: leve; G2: 4,0-7,9: moderada e G3: 8,0-10: intensa) as participantes que possuíam os menores escores de dor (G1) possuíam melhor EV em relação aos demais grupos (tabela 1).

Tabela 1 - Comparação entre os 3 grupos de acordo com a percepção de dor (n=55).

\begin{tabular}{ccccc}
\hline & G1 $(\mathbf{n}=\mathbf{3 1})$ & $\mathbf{G} 2(\mathbf{n}=\mathbf{4 8})$ & $\mathbf{G 3}(\mathbf{n}=\mathbf{3 1})$ & $\mathbf{p}$ \\
Escore FANTASTICO & $74,2 \pm 6,8^{\mathrm{a}}$ & $68,9 \pm 10,3^{\mathrm{b}}$ & $69,6 \pm 7,4^{\mathrm{b}}$ & $<0,005$ \\
\hline
\end{tabular}

EVA: escala visual analógica, letras diferentes indicam diferenças estatisticamente significantes. G- grupos e intensidades de dor avaliada pela Escala Visual Analógica: G1: 0,0-3,9: leve; G2: 4,0-7,9: moderada e G3: 8,0-10: intensa

\section{DISCUSSÃO}

O objetivo deste estudo foi relacionar a intensidade da dor referida durante o período folicular do ciclo menstrual e o estilo de vida adotado por universitárias. Ao dividir o grupo de acordo com a intensidade de dor, percebe-se que aquelas com menor dor referida são aquelas que adotam um melhor estilo de vida.

O instrumento adotado neste estudo para avaliar o estilo de vida foi o FANTASTICO, um instrumento validado no Brasil em português ${ }^{9}$, além disso, a EVA é uma forma amplamente utilizada para mensurar a percepção de dor ${ }^{8,11}$.

Embora fosse esperado que houvesse associação entre o estilo de vida e a dor referida durante o período folicular das universitárias, isto não foi observado no presente estudo. Contudo, ao dividir as participantes de acordo com a intensidade da dor, observou-se diferença significante. Mulheres que sentem menor dor, possuem melhor estilo de vida.

Sabe-se que um estilo de vida saudável pode interferir na dor durante o ciclo menstrual das mulheres, pois o estilo de vida é algo controlável e mutável relacionado aos comportamentos adotados que podem afetar positiva ou negativamente a saúde dos indivíduos ${ }^{12}$.

Ao comparar o estilo de vida de mulheres com e sem dismenorreia, um estudo verificou diferenças significantes 
em relação ao comportamento alimentar, atividade física, estresse e relações sociais, contudo, não foram observadas diferenças em relação ao autocuidado, tabagismo, bebidas e uso de drogas ${ }^{12}$. Os autores concluíram que para prevenir e reduzir a incidência de dismenorreia primária, conhecimento e conscientização devem ser estimulados em mulheres jovens por meio da educação sobre o estilo de vida ${ }^{12}$. Ainda, outro estudo demonstrou que a atividade física é eficaz na redução da dor decorrente da dismenorreia, podendo assim ser uma forma de tratamento adjuvante dessa condição ${ }^{8}$.

Neste estudo, embora as participantes não tenham sido questionadas quanto à presença de dismenorreia, não foi observada associação entre dor referida e estilo de vida, contudo ao serem classificadas e comparadas quanto a intensidade da dor, observou-se que aquelas com menor dor referida no período folicular, possuíam melhor estilo de vida, ou seja, EV considerado "muito bom". Já aquelas que referiam dor moderada ou intensa, possuíam estilo de vida considerado "bom", em média.

Sabe-se que na fase folicular do ciclo menstrual há maior sensibilidade à dor ${ }^{4}$, e que as mulheres que possuem um estilo de vida considerado saudável possuem menor dor, confirmando a influência do EV na percepção de dor durante o período menstrual. Dores menstruais podem causar vários prejuízos para as mulheres, incluindo deficiência no funcionamento físico, impacto na saúde psicológica e disfunção relacionadas a aspectos sociais e ocupacionais ${ }^{13}$.

Um fator limitante neste estudo, é a falta de padronização de avaliação da dor durante a fase mais dolorosa do ciclo menstrual, ou seja, a folicular, contudo, foi solicitado a participante que lembrasse do último cilco menstural e apontasse como foi a sua dor.

Um fato que merece destaque neste estudo diz respeito ao instrumento de EV, que não avalia profundamente fatores como hábitos alimentares e prática exercício físico. Esses fatores podem exercer influência importante na presença e percepção da dor. Assim, futuros estudos que avaliem esses aspectos detalhadamente devem ser realizados. Ainda, cabe ressaltar que instrumentos mais objetivos de avaliação da dor no período folicular, como a algometria, devem ser empregados por estudos futuros.

\section{CONSIDERAÇÕES FINAIS}

Não foi encontrada associação entre a intensidade da dor durante o período folicular e o estilo de vida adotados pelas universitárias participantes deste estudo. Apesar disso, ao dividir o grupo de acordo com a intensidade da dor, notou-se que aquelas com menor dor referida possuem um melhor estilo de vida. 


\section{REFERÊNCIAS}

1. Merskey H, Bogduk N. Classification of chronic pain: descriptions of chronic pain syndromes and definitions of pain terms. 2a. ed. Seattle: International Association for Study of Pain Press, 1994.

2. Lira MOSC, Carvalho MFAA. Dor aguda e relação de gênero: diferentes percepções em homens e muIheres. Rev Rene. 2013; 14(1):71-81.

3. Kazama M, Maruyama K, Nakamura K. Prevalence of dysmenorrhea and its correlating lifestyle factors in Japanese female junior high school students.Tohoku J Exp Med 2015;236(2):107-13.

4. Alfieri FM, Bernardo KMA, Pinto YS, Silva NCOV, Portes LA. Pain tolerance and cardiorespiratory fitness in women with dysmenorrhea. Rev Dor 2017; 18: 311-315.

5. Bavil AB, Dolatina M, Mahmmodi Z, Baghan AA. Comparison of lifestyles of young women with and without primary dysmenorrhea. Electronic Physician 2016; 8 (3): 2107-2114.

6. Portes LA. Estilo de Vida e Qualidade de Vida: semelhanças e diferenças entre os conceitos. Lifestyle J 2011;1(1):8-10.

7. Bianco V, Cestari AM, Casati D, Cipriani S, Radici G, Valente I. Premenstrual syndrome and beyond: lifestyle, nutrition, and personal facts. Minerva Ginecol 2014;66(4):365-75.

8. Quintana LM, Heinz LN, Portes LA, Alfieri FM. Influência do nível de atividade física na dismenorréia. Revista Brasileira de Atividade Física e Saúde. 2010; 15:101-104.

9. An\z CRR, Reis RS, Petroski EL. Versão brasileira do questionário "Estilo de Vida Fantástico": tradução e validação para adultos jovens. Arq Bras Cardiol 2008;2(91):102-09.

10. Chapman RS, Syrjala KL. Measurement of pain. In: Bonica JJ, eds. The management of pain. Londres, Lea \&Febiger, 1990, 580-594. 
11. Drummond JP. Dor aguda: fisiopatologia, clínica e terapêutica. São Paulo: Atheneu, 2000.

12. Sharma R, Biedenharn KR, Fedor JM, Agarwal A. Lifestyle factors and reproductive health: taking control of your fertility. Reprod Biol Endocrinol 2013;16;11:66.

13. Biggs WS, Demuth RH. Premenstrual syndrome and premenstrual dysphoric disorder. Am Fam Physician 2011;15;84(8):918-24. 